Apidologie, 1981, 12 (4), 329-343.

\title{
IN WELCHEM ABSCHNITT DES PAARUNGSVERHALTENS DER BIENENKÖNIGIN FINDET DIE INDUKTION DER EIABLAGE STATT?
}

\author{
Gudrun KoENIGER \\ Institut für Bienenkunde (Polytechnische Gesellschaft), \\ Fachbereich Biologie der J. W. Goethe-Universität Frankfurt a.M., \\ Im Rothkopf 5, 6370 Oberursel/Ts., F.R.G.
}

\section{ZUSAMMENFASSUNG}

Das Paarungsverhalten der Bienenkönigin wurde durch experimentelle Eingriffe in verschiedene Abschnitte unterteilt. Dadurch konnte ihre Bedeutung für die Induktion der Eiablage einzeln untersucht werden.

1. Der aktive Flug der Königin bewirkte weder eine Entwicklung der Ovarien noch den Beginn der Eiablage.

2. Auch die Verfolgung durch paarungsbereite Drohnen allein blieb ohne Wirkung.

3. Die Umklammerung durch Drohnen ohne nachfolgende Verhängung hatte nur einen geringen Einfluss.

4. Eine Verhängung von freifliegenden, paarungsbereiten Drohnen mit fixierten Königinnen bewirkten bei $68 \%$ der Königinnen den Beginn der Eiablage, obwohl die Eversion des Endophallus unvollständig blieb und kaum Sperma oder Mucus übertragen wurde. Das gleiche Ergebnis wurde erreicht, wenn jeglicher äusserer Kontakt zwischen Drohnen und Königinnen durch eine Plastikröhre verhindert wurde.

5. Verhängungen von Königinnen und Drohnen im Labor mit künstlich zur Eversion gebrachten Endophalli zeigten keine Wirkung. Allerdings waren diese Verhängungen viel lockerer als bei aktiven Drohnen.

Nach diesen Ergebnissen muss der entscheidende Reiz während der Verhängung in der Stachelkammer und Bursa copulatrix perzipiert werden. Dieser Reiz ist unabhängig von der Übertragung des Begattungszeichens, vom aktiven Flug und vom äusseren Kontakt.

\section{EINLEITUNG}

Natürlich gepaarte Königinnen beginnen bereits 12-24 Stunden nach dem letzten erfolgreichen Hochzeitsflug mit der Eiablage (ALBER et al., 1955). Künstlich besamte Königinnen dagegen beginnen erst nach Wochen Eier zu legen, wie es auch von 
unbegatteten Königinnen bekannt ist, obwohl die Spermatheken wie nach der natürlichen Paarung prall mit Spermien gefüllt sind. Eine frühere Eiablage nach der künstlichen Besamung wird in der Praxis durch eine zweimalige $\mathrm{CO}_{2}$-Narkose erreicht (MACKENSEN, 1947). Welche Faktoren im Ablauf der natürlichen Paarung die Eiablage induzieren, ist noch nicht nachgewiesen. In diesem Zusammenhang wurde schon oft über die Bedeutung des Fluges oder des Begattungszeichens diskutiert. Das Begattungszeichen, das aus dem Sekret der männlichen Anhangsdrüsen (Mucus) und Teilen des Endophallus besteht, verschliesst die Stachelkammer einer frisch gepaarten Königin für einige Stunden. $\mathrm{Da}$ von vielen anderen Insekten bekannt ist, dass Substanzen aus dem Sekret der männlichen Anhangsdrüsen die Eiablage induzieren, war zunächst die Bedeutung des Begattungszeichens untersucht worden. Sein Einfluss hatte sich als sehr gering erwiesen (KoENiger G., 1976).

In den hier vorgelegten Versuchen wurden deshalb andere Abschnitte des Paarungsverhaltens untersucht. Dabei wurde das gleiche Prinzip angewandt wie bei den Untersuchungen des Begattungszeichens; zuerst wurde der Einfluss eines Abschnittes für sich allein geprüft, danach wurde dieser Abschnitt, soweit es möglich war, einzeln aus dem Paarungsablauf ausgeschaltet.

Folgende Abschnitte des Paarungsverhaltens konnten auf diese Weise untersucht werden :

1. Aktiver Flug der Königin:

a) Bewirkt der aktive Flug ohne Kontakt zu Drohnen die Ovarentwicklung und die Eiablage?

b) Ist der aktive Flug eine der notwendigen Voraussetzungen zur Induktion der Eiablage?

2. Drohnenkontakte mit der Königin:

a) Hat der visuelle und olfaktorische Kontakt mit paarungsbereiten Drohnen einen Einfluss auf Ovarentwicklung und Eiablage?

b) Haben die rein äusserlichen Berührungsreize durch die Drohnen einen Einfluss auf Ovarentwicklung und Eiablage?

c) Sind die äusseren Kontakte eine der notwendigen Voraussetzungen zur Induktion der Eiablage?

d) Sind die Verhängungen in der Stachelkammer eine der notwendigen Voraussetzungen zur Induktion der Eiablage?

\section{MATERIAL UND METHODE}

Für die Versuche wurden Königinnen verschiedener Rassen von Apis mellifera (A.m. carnica, A.m. ligustica, A.m. capensis) benutzt. Alle Königinnen wurden in kleinen Begattungskästen mit etwa 2000 Arbeiterinnen gehalten. Im allgemeinen wurden die Versuche mit Königinnen im Alter von 8 bis 14 Tagen 
durchgeführt. Der Beginn der Eiablage wurde täglich kontrolliert. Wenn die Königinnen 21 oder höchstens 23 Tage alt waren, wurde das Gewicht der Ovarien bestimmt und die Spermatheka auf ihren Gehalt an Spermien untersucht.

Die Anzahl und Dauer der Ausflüge der Königinnen wurde mit Hilfe eines kleinen Flugtunnels kontrolliert (ALBERT et al., 1955). Ein Ausbleiben von mehr als $15 \mathrm{~min}$ wurde als Hochzeitsflug gewertet.

\section{Ausflüge ohne Kontakt zu Drohnen}

Die Königinnen wurden mit ihren Völkchen auf die Nordseeinsel Norderney transportiert. Zuvor waren alle anderen Völker (und damit auch alle Drohnen) von dort entfernt worden. Norderney wird seit vielen Jahren mit gutem Erfolg als Belegstelle benutzt. Den Königinnen wurde demnach eine Umgebung geboten, die für Hochzeitsflüge geeignet ist.

\section{Verhinderung der Flugaktivität der Königin auf dem Drohnensammelplatz}

a) Die Königinnen wurden in kleine Käfige aus Drahtgeflecht gesperrt und mit einem Ballon auf dem Drohnensammelplatz in eine Höhe zwischen 15 und $25 \mathrm{~m}$ gebracht. Wenn sie während einer Zeit von $15 \mathrm{~min}$ stark von Drohnen verfolgt worden waren, wurden sie wieder in ihr Volk zurückgegeben.

\section{Kopulationsversuche mit fixierten Königinnen}

b) Um einen direkten Kontakt zwischen Königin und Drohnen zu ermöglichen, wurden die Königinnen längs an einen dünnen Draht $(\varnothing=0,1 \mathrm{~mm})$ geklebt und zwar an drei Stellen : Am Scutum des Thorax, am ersten und am vorletzten Tergit des Abdomens (Abb. 1). Die Fixierung des Abdomens erwies sich als notwendig, da Königinnen, die nur am Thorax festgeklebt sind, ihren Hinterleib im allgemeinen herunterhängen lassen und so den Drohnen ein Aufreiten unmöglich machen. Mit Hilfe des Drahtes aber kann man das Abdomen in die Flugstellung biegen.

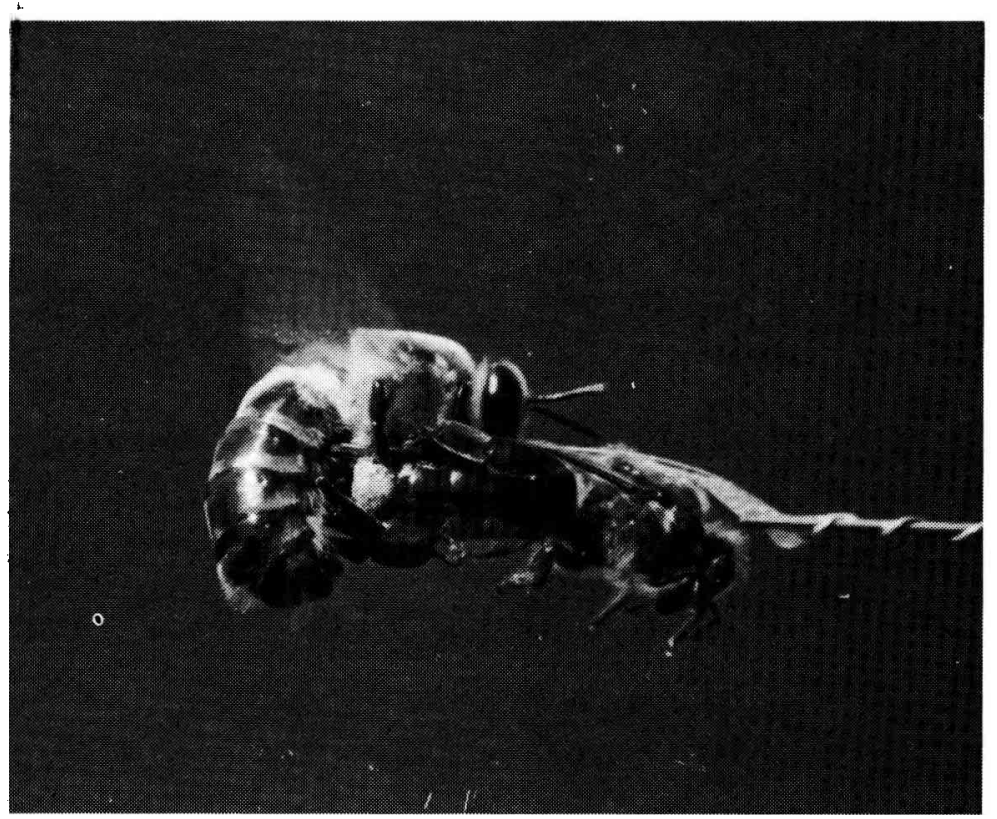

Aвв. 1. - Die Königin wurde mit Thorax und Abdomen an einen dünnen Draht geklebt. FIG. 1. - Thorax and abdomen of the queen glued to a thin wire. 
Der dünne Draht wurde am frontalen Ende über einen festen Draht gewickelt. Der feste Draht wurde an einen $1.5 \mathrm{~m}$ langen Stab gesteckt, der dann mit einem Teleskopmast in die Höhe von $8 \mathrm{~m}$ gebracht wurde (KoEniger, G. et al.; 1979). Dort wurde die Königin mit einer Geschwindigkeit von 7-14 km/h auf einer Kreisbahn bewegt. Dadurch war es den Drohnen möglich, die Königinnen aus der richtigen Position, nämlich von hinten gegen den Wind, anzufliegen.

\section{Künstliche Öffnung der Stachelkammer}

Die Königinnen wurden ohne $\mathrm{CO}_{2}$-Narkose in einen leıcht modifizierten Besamungsapparat eingespannt (KoENIGER G., 1976). Während die Stachelkammer durch eine Pinzette und einen Haken offen gehalten wurde, wurden die Gelenke zwischen dem letzten Tergit und Sternit verklebt (Klebstoff : Pattex), so dass die Königin die Stachelkammer für die nächsten 30 Stunden nicht verschliessen konnte. Innerhalb der nächsten 2-4 Tage löste sich der Klebstoff langsam wieder von der Königin.

\section{Verhinderung von äusseren Kontakten bei Verhängungen mit Drohnen}

Die Königinnen wurden in kleine Plastikröhren gesteckt. Nur der Kopf, die Flügel und die Beine waren frei. An diesen Stellen werden die Königinnen während der Paarung von den Drohnen nicht berührt (KoENiger G. et al., 1979). Die Röhre wurde am Scutum des Thorax festgeklebt. Danach wurden Tergit und Sternit des letzten Segmentes so an die hintere Öffnung der Röhre geklebt, dass die Stachelkammer weit geöffnet blieb (Abb. 2). Auf diese Weise konnten sich die Drohnen mit der Königin verhängen, ohne sie äusserlich zu berühren. Zu beachten war. dass die Röhre sich eng um das Abdomen schloss. da sonst die Drohnen den Endophallus in den Raum zwischen Abdomen und Röhre evertierten.

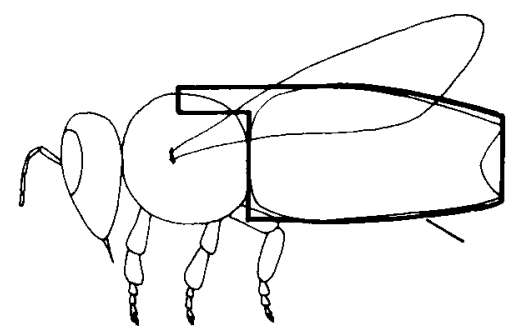

Авв. 2. - Königin in der Plastikröhre.

FIG. 2. - Abdomen of the queen covered by a plastic tube.

\section{ERGEBNISSE}

\section{Einfluss des aktiven Fluges der Königin auf den Beginn der Eiablage}

a) Aktiver Flug ohne Kontakt zu Drohnen

Am 28-5-1976 wurden 30 Königinnen auf der Insel Norderney aufgestellt. Bis zum 4.6. herrschten für die Jahreszeit ungewöhnlich kalte Temperaturen, so dass die Königinnen erst in einem Alter von 14 bis 17 Tagen ausfliegen konnten. Die Anzahl der Ausflüge war sehr hoch, sie lag im Mittel bei 5,6 pro Königin. 12 Königinnen blieben jedes Mal nur für kurze Zeit fort, 11 dagegen blieben länger als 15 min ("Hochzeitsflug »). Die restlichen 7 Königinnen gingen verloren. Zur gleichen Zeit wurde in Oberursel 4 Königinnen ein normaler Hochzeitsflug ermöglicht. 
Keine der Norderney-Königinnen begann bis zum Alter von 22 Tagen mit der Eiablage (Tab. 1). Die Ovarien hatten ein Gewicht zwischen 7 und $11 \mathrm{mg}$, es hatte noch kein Reifungsprozess eingesetzt. Die 4 Kontrollköniginnen dagegen legten Eier, die Ovargewichte betrugen zwischen 50 und $70 \mathrm{mg}$.

TAB. 1. - Ovargewichte von Königinnen im Alter von 22 Tagen, deren Hochzeitsflüge in drohnenfreiem Gebiet stattfanden.

TABL. 1. - Influence on ovaries of flights on an island without drones.

\begin{tabular}{|c|c|c|c|}
\hline & n $P Q$ & $\begin{array}{l}\text { Ovarg } \\
\text { Weight }\end{array}$ & $\begin{array}{l}\text { wicht }(\mathrm{mg}) \\
\text { fovaries }(\mathrm{mg})\end{array}$ \\
\hline $\begin{array}{l}\text { \$ } 9 \text { mit normalem Hochzeitsflug } \\
\text { \& with normal mating flight }\end{array}$ & 4 & $50-70$ & $\begin{array}{l}\text { Eiablage } \\
\text { Oviposition }\end{array}$ \\
\hline $\begin{array}{l}\text { Ausflug }<15 \mathrm{~min} \\
\text { Flight }\end{array}$ & 12 & $7-11$ & \\
\hline $\begin{array}{l}\text { 1 Ausflug }>15 \mathrm{~min} \\
\text { 1 flight }\end{array}$ & 3 & $7-11$ & \\
\hline $\begin{array}{l}2 \text { Ausflüge }>15 \mathrm{~min} \\
2 \text { flights }\end{array}$ & 7 & $7-11$ & \\
\hline $\begin{array}{l}3 \text { Ausflüge }>15 \mathrm{~min} \\
3 \text { flights }\end{array}$ & 1 & $7-11$ & \\
\hline $\begin{array}{l}\text { Qqgesperrt } \\
\text { Qqconfined }\end{array}$ & 23 & 9.7 & \\
\hline
\end{tabular}

b) Ausschluss des aktiven Fluges der Königin aus dem Paarungsablauf

In den Sommern 1978 und 1979 wurden 38 Königinnen an einem Draht fixiert und den Drohnen auf dem Drohnensammelplatz in einer Höhe von $8 \mathrm{~m}$ zur Paarung geboten. Diese Könginnen zeigten nur selten für einige Sekunden Flugversuche. Sie waren auch nicht in Paarungsstimmung, denn sie öffneten ihre Stachelkammern nicht, so dass diese künstlich durch Verklebung der Gelenke offen gehalten werden mussten. Ausserdem versuchten sie fortwährend, die aufreitenden Drohnen mit den Hinterbeinen abzuwehren. Trotzdem gelang es den Drohnen sich mit ihnen zu verhängen, aber dabei kam es nur in Ausnahmefällen zur vollständigen Eversion des Endophallus. Gewöhnlich kam die Eversion in Höhe der Cervix zum Stillstand, wie es auch bei den Chloroform-Narkosen geschieht. Dadurch gelangten bei dieser Art der Verhängung keine oder wenige (bis zu 5000 ) Spermatozoen in die Spermatheka (das entspricht $0 \%$ bzw. $5 \%$ der normalen Menge). Da der Mucus erst nach den Spermien ausgeschieden wird, konnte er vermutlich nicht in die Königin übertragen werden. Die Verhängung selbst aber war so fest, dass die Drohnen sich nicht allein von den Königinnen lösten. Um eine Trennung ohne Verletzung der Königinnen zu erreichen, wurde der Endophallus in vielen Fällen in Höhe des Vestibulums angeschnitten. 
Nach vielen Versuchen, bei denen die Königinnen nach der Fixierung am Mast und 5-8 Verhängungen mit Drohnen innerhalb der nächsten 10 Tage starben, gelang es schliesslich die Methode so weit zu verbessern, dass $24 \mathrm{Königinnen} \mathrm{über} \mathrm{einen} \mathrm{längeren}$ Zeitraum beobachtet werden konnten. 15 von ihnen (64\%) begannen in einem Alter zwischen 15 und 21 Tagen Eier zu legen (Tab. 2). Die 10 Kontrollköniginnen, die mit

ТАВ. 2. - Ovargewichte und Beginn der Eiablage von Königinnen ohne aktiven Flug, aber mit 5-8 Verhängungen mit Drohnen.

TABL. 2. - Influence of mating without active flight on ovaries and oviposition.

\begin{tabular}{|c|c|c|c|}
\hline & 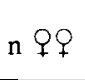 & $\begin{array}{l}\text { nq in Eiablage } \\
\text { n?Q ovipositing }\end{array}$ & $\begin{array}{c}\mathrm{n} 99 \text { mit Ovargewicht }>20 \mathrm{mg} \\
\mathrm{n} 99 \text { with ovaries }>20 \mathrm{mg}\end{array}$ \\
\hline $\begin{array}{l}\text { Versuchs } q Q \\
\text { Tested } Q Q\end{array}$ & 24 & $15(64 \%)$ & $15(64 \%)$ \\
\hline $\begin{array}{l}\text { Kontroll- } 9 Q \\
\text { Control } 99\end{array}$ & 10 & - & - \\
\hline
\end{tabular}

geöffneter Stachelkammer ebenfalls an einen Draht geklebt wurden, wurden ohne auf den Mast zu kommen wieder in ihre Völkchen gesetzt. Keine von ihnen legte Eier oder entwickelte ihre Ovarien.

Eine Beziehung zwischen Anzahl der Spermatozoen in der Spermatheka und dem Beginn der Eiablage lässt sich nicht feststellen. So hatten z.B. 3 der legenden Königinnen überhaupt keine, 2 der nicht legenden dagegen 5000 Spermatozoen. Im Durchschnitt waren bei den legenden Königinnen etwa 1000 , bei den nicht legenden etwa 2000 Spermatozoen in die Spermatheka gelangt.

2. Einfluss von verschiedenen Kontakten der Königin mit Drohnen auf den Beginn der Eiablage

a) Visueller und olfaktorischer Kontakt

Durch Einsperren in kleine Drahtkäfige wurden 13 Königinnen auf dem Drohnensammelplatz vor dem direkten Kontakt mit paarungsbereiten Drohnen geschützt. Keine dieser Königinnen legte Eier, 2 wiesen Ovarien mit einem Gewicht über $20 \mathrm{mg}$ auf.

b) Direkte, aber rein äusserliche Kontakte mit Drohnen

Für diese Versuche wurden 2 Gruppen von Königinnen verschieden behandelt : die erste Gruppe wurde nur an den Draht geklebt und am Mast den Drohnen geboten. Da diese Königinnen ihre Stachelkammern nicht öffnen, war den Drohnen nur ein Greifen und Aufreiten möglich. Der zweiten Gruppe wurden die Stachelkammern mit Klebstoff gänzlich verschlossen. Danach konnten sie frei zur Paarung ausfliegen. Von der ersten Gruppe konnten 16 und von der zweiten Gruppe 12 Königinnen ausgewertet 
werden (Tab. 3). Insgesamt begannen 5 (18\%) von ihnen mit der Eiablage, 7 (24\%) hatten Ovargewichte von mehr als $20 \mathrm{mg}$. Von 14 Kontrollköniginnen, die nicht ausgeflogen waren, legte keine Eier, 2 (14\%) hatten entwickelte Ovarien. 2 Königinnen hatten nur Orientierungsflüge unternommen, die aber keinen Effekt zeigten.

ТАВ. 3. - Einfluss von direkten, rein äusserlichen Kontakten von Königinnen und Drohnen auf die Ovarentwicklung und die Eiablage.

TABL. 3. - Influence of external contact on ovaries and oviposition.

\begin{tabular}{|c|c|c|c|}
\hline & n 99 & $\begin{array}{l}\text { n } Q Q \text { in Eiablage } \\
\text { n } \$ Q \text { ovipositing }\end{array}$ & $\begin{array}{c}\mathrm{n} q 9 \text { mit Ovargewicht }>20 \mathrm{mg} \\
\mathrm{n} q q \text { with ovaries }>20 \mathrm{mg}\end{array}$ \\
\hline $\begin{array}{l}\text { Qqam Mast } \\
\text { qon mast }\end{array}$ & 16 & $2(17 \%)$ & $2(17 \%)$ \\
\hline $\begin{array}{l}\text { Ausflug }>15 \mathrm{~min} \\
\text { Flight }\end{array}$ & 12 & $3(19 \%)$ & $5(31 \%)$ \\
\hline $\begin{array}{l}\text { Ausflug }<15 \mathrm{~min} \\
\text { Flight }\end{array}$ & 2 & - & - \\
\hline $\begin{array}{l}\text { QQgesperrt } \\
\text { \$9 confined }\end{array}$ & 14 & - & $2(14 \%)$ \\
\hline
\end{tabular}

c) Ausschluss der äusserlichen Kontakte während der Verhängung der Drohnen in der Stachelkammer

Bei dem letzten Ergebnis erhob sich die Frage, ob die äusseren Kontakte nur einen geringen Einfluss auf den Beginn der Eiablage haben, oder ob sie zusammen mit den Verhängungen eine wichtige Rolle spielen. Deshalb wurden die Abdomina von 23 Königinnen mit einer Plastikröhre bedeckt (Abb. 2), die Stachelkammer aber für die Drohnen frei zugänglich gehalten. Die Königinnen waren für die Drohnen nicht mehr so attraktiv wie mit freiem Abdomen, was sich mit der Verdeckung der Tergittaschendrüsen (RENNER und VIERLING, 1977) erklären lässt. Trotzdem kam es bei 7 Königinnen zu jeweils 5-8 Verhängungen. Bei 5 weiteren Königinnen war offensichtlich der Zwischenraum zwischen Röhre und Abdomen zu gross, denn die Drohnen kopulierten in diesen Raum hinein, wobei die Eversion häufig vollständig war. Von den restlichen Königinnen wurde ein Teil als Kontrolle mit abgedecktem Abdomen auf den Mast gebracht, aber nicht von Drohnen beflogen, der andere Teil nur mit einer Röhre beklebt und danach wieder in sein Volk gesetzt. Von den 7 Königinnen mit Verhängungen begannen fünf Eier zu legen (Tab. 4). Dagegen hatten weder die Kopulationen in den Zwischenraum noch die Beklebung der Königinnen allein einen Effekt auf die Eiablage. Nur zwei von ihnen wiesen ein Ovargewicht von $20 \mathrm{mg}$ auf

3. Experimentelle Einführung des halb evertierten Endophallus in die Stachelkammer (künstliche Verhängung)

Bei 30 Königinnen wurden die Stachelkammern durch Klebstoff offen gehalten. In der ersten Versuchsserie wurden die Drohnen mit Chloroform betäubt, bis der 
ТАВ. 4. - Ovargewichte und Eiablage von Königinnen mit 5-8 Verhängungen bei abgedeckten Abdomina.

TABL. 4. - Influence of mating without active flight and without external contacts.

\begin{tabular}{|c|c|c|c|}
\hline & $n q Q$ & $\begin{array}{l}\text { n } Q Q \text { in Eiablage } \\
\text { nœQ ovipositing }\end{array}$ & $\begin{array}{c}\mathrm{n}+9 \text { mit Ovargewicht }>20 \mathrm{mg} \\
\mathrm{n} q 9 \text { with ovaries }>20 \mathrm{mg}\end{array}$ \\
\hline $\begin{array}{l}\text { Verhängung in } 9 Q \\
\text { Insertion in } 9 q\end{array}$ & 7 & $5(71 \%)$ & $5(71 \%)$ \\
\hline $\begin{array}{l}\text { Verhängung zwischen Röhre und } \\
\text { Abdomen } \\
\text { Insertion between } q q \text { and tube }\end{array}$ & 5 & 0 & 0 \\
\hline $\begin{array}{l}\text { IQnur in der Röhre } \\
\text { qQ in tubes only }\end{array}$ & 11 & 0 & $2(18 \%)$ \\
\hline $\begin{array}{l}\text { QYgesperrt } \\
\text { Qq confined }\end{array}$ & 3 & 0 & 0 \\
\hline
\end{tabular}

Endophallus zur Hälfte evertiert war. In der zweiten Serie wurde der Endophallus durch Druck auf den Thorax des Drohnes zur Eversion gebracht. Dann wurde der Endophallus in die Stachelkammer eingeführt. Danach wurden Königin und Drohn in der Paarungsstellung aufeinander gehalten, wobei vor allem darauf geachtet wurde, dass auch die Hinterbeine des Drohnes das Abdomen der Königin berührten, sie wurden dabei mit der Pinzette geführt, um sie mehrmals am Abdomen lang streichen zu können. Bei diesen Versuchen gelang es jedoch nicht, den Endophallus so stark aufzublähen, wie die Drohnen es selbst tun, obwohl es durch weiteren Druck auf das Abdomen versucht wurde. Meist wurde dadurch nur die vollständige Eversion des Endophallus erreicht. So waren die Verhängungen nie so fest wie bei den Versuchen am Mast. Nach 10 künstlichen Verhängungen wurden die Königinnen wieder in ihr Volk zurückgesetzt. 24 von ihnen blieben ohne Infektion und konnten ausgewertet werden. Nur eine von ihnen begann Eier zu legen, 4 wiesen ein Ovargewicht von mehr als $20 \mathrm{mg}$ auf (Tab. 5). Die 9 Kontrollköniginnen waren im Stock gesperrt, von ihnen hatten 2 entwickelte Ovarien.

\footnotetext{
TAB. 5. - Ovarentwicklung und Eiablage von Königinnen nach 10 künstlichen Verhängungen.
}

TABL. 5. - Influence of manual insertion of endophalli on ovary development and oviposition.

\begin{tabular}{|c|c|c|c|}
\hline & n 99 & $\begin{array}{l}\text { n } q 9 \text { in Eiablage } \\
\text { nq9ovipositing }\end{array}$ & $\begin{array}{c}\text { n } 9 \text { mit Ovargewicht }>20 \mathrm{mg} \\
\text { n } 9 \text { qwith ovaries }>20 \mathrm{mg}\end{array}$ \\
\hline $\begin{array}{l}\text { Versuchs } q Q \\
\text { Tested } q 9\end{array}$ & 24 & $1(4 \%)$ & $4(16 \%)$ \\
\hline $\begin{array}{l}\text { Kontroll- } Q Q \\
\text { Control } Q q\end{array}$ & 9 & 0 & $2(22 \%)$ \\
\hline
\end{tabular}




\section{DISKUSSION}

Im Verlauf dieser Arbeit sollte zunächst herausgefunden werden, welche Abschnitte der Paarung für die Induktion der Eiablage wichtig sind. Erst nach dieser Eingrenzung sind gezielte Versuche möglich, die Aufschluss über die Art des Reizes geben können. Dabei wurde im Prinzip folgende Methode angewandt : Zunächst wurde der Einfluss von einzelnen Abschnitten für sich allein untersucht. Danach wurde der reziproke Ansatz angewendet; es wurde nach Möglichkeit nur dieser bestimmte Abschnitt gezielt ausgeschaltet. Folgende Einzelelemente des Paarungsverhaltens wurden auf diese Weise untersucht :

I. Behandlung der jungen Königin im Stock durch die Arbeiterinnen;

II. Aktiver Flug der Königin;

III. Verfolgung durch die Drohnen (visueller und olfaktorischer Kontakt);

IV. Umklammerung durch paarungsbereite Drohnen (äusserlicher Kontakt);

V. Einführung des halb evertierten Endophallus;

VI. Wirkung der Sekrete der männlichen Anhangsdrüsen.

\section{(Zusammenfassung aller Daten siehe Tabelle 6).}

Damit eine Königin überhaupt zur Paarung ausfliegt, muss sie mit so vielen Arbeiterinnen zusammen gehalten werden, dass der Aufbau einer Volkseinheit möglich ist. Bei allen Versuchen wurde mit etwa 2000 Arbeiterinnen pro Königin gearbeitet, eine Methode, die seit Jahrzehnten von Imkern mit Erfolg angewandt wird. Somit waren alle Kontroll- und Versuchsköniginnen von Arbeiterinnen gepflegt. Königinnen ohne Arbeiterinnen sind nur wenige Tage lebensfähig. Die Pflege durch Arbeiterinnen ist also eine Voraussetzung sowohl für den Hochzeitsflug als auch für eine Eiablage, aber sie hat keinen direkten Einfluss auf die Induktion der Eiablage bei der jungfräulichen Königin, denn von 100 Königinnen, deren Ausflug verhindert wurde, begann nur eine Eier zu legen (Tab. 6, I).

Der aktive Flug der Königin allein erwies sich als wirkungslos auf die Ovarentwicklung und auf den Beginn der Eiablage (Tab. 6, II). Dieses Ergebnis stimmt mit den Versuchen von PEER (1957) über Flugweiten von Königinnen überein, bei denen sich zeigte, dass Königinnen bis zu einem Alter von 30 Tagen wiederholt ausfliegen, wenn sie nicht mit Drohnen zusammentreffen. Für den reziproken Versuch, ob der Flug der Königin als additiver Reiz für die Eiablage notwendig ist, wurde fixierten Königinnen eine Verhängung mit paarungsbereiten, freifliegenden Drohnen ermöglicht. Bei dieser Paarung stoppte die Eversion des Endophallus fast immer in Höhe der Cervix, was vielleicht auf die Notwendigkeit einer aktiven Beteiligung der Königin hinweist. Trotzdem begannen $64 \%$ dieser fixierten Königinnen Eier zu legen. Demnach kann der aktive Flug, wenn überhaupt, nur einen geringen Einfluss auf den Beginn der Eiablage haben (Tab. 6, II). 
TAB. 6. - Zusammenfassung aller bekannter Daten.

TABL. 6. - Summary of dates.

Das Paarungsverhalten wurde in folgende Abschnitte unterteilt :

The mating process was divided into the following segments :

\begin{tabular}{|c|c|c|c|c|c|c|}
\hline$\alpha$ & $\beta$ & $v$ & $\delta$ & $\varepsilon$ & $\zeta$ & $\eta$ \\
\hline $\begin{array}{l}99 \text { mit } \not \varnothing\} \\
\text { im Stock }\end{array}$ & $\begin{array}{l}\text { Aktiver } \\
\text { Flug der } \$ Q \\
\\
\text { Active } \\
\text { flight of } \\
\text { the } \$ 9\end{array}$ & $\begin{array}{l}\text { Verfolgung } \\
\text { durch } \sigma^{\top} \sigma^{7} \\
\text { By means } \\
\text { of } \delta \delta^{\star} \text { pursuit }\end{array}$ & $\begin{array}{l}\begin{array}{l}\text { Greifen } \\
\text { durch } \sigma^{7} \sigma^{*}\end{array} \\
\text { By means } \\
\text { of } \sigma^{*} \sigma^{*} \text { touch }\end{array}$ & $\begin{array}{l}\text { Unvollständ. } \\
\text { Eversion des } \\
\text { Endophallus } \\
\text { in die Stachel- } \\
\text { kammer } \\
\text { Incomplete } \\
\text { eversion } \\
\text { of endoph. } \\
\text { into sting } \\
\text { chamber }\end{array}$ & $\begin{array}{l}\text { Injektion } \\
\text { von Mucus } \\
\text { und ander. } \\
\text { Sekreten } \\
\text { Injection } \\
\text { of mucous } \\
\text { or other } \\
\text { secretion }\end{array}$ & $\begin{array}{l}\text { Injektion } \\
\text { von } \\
\text { Sperma } \\
\text { Injection } \\
\text { of sperm }\end{array}$ \\
\hline
\end{tabular}

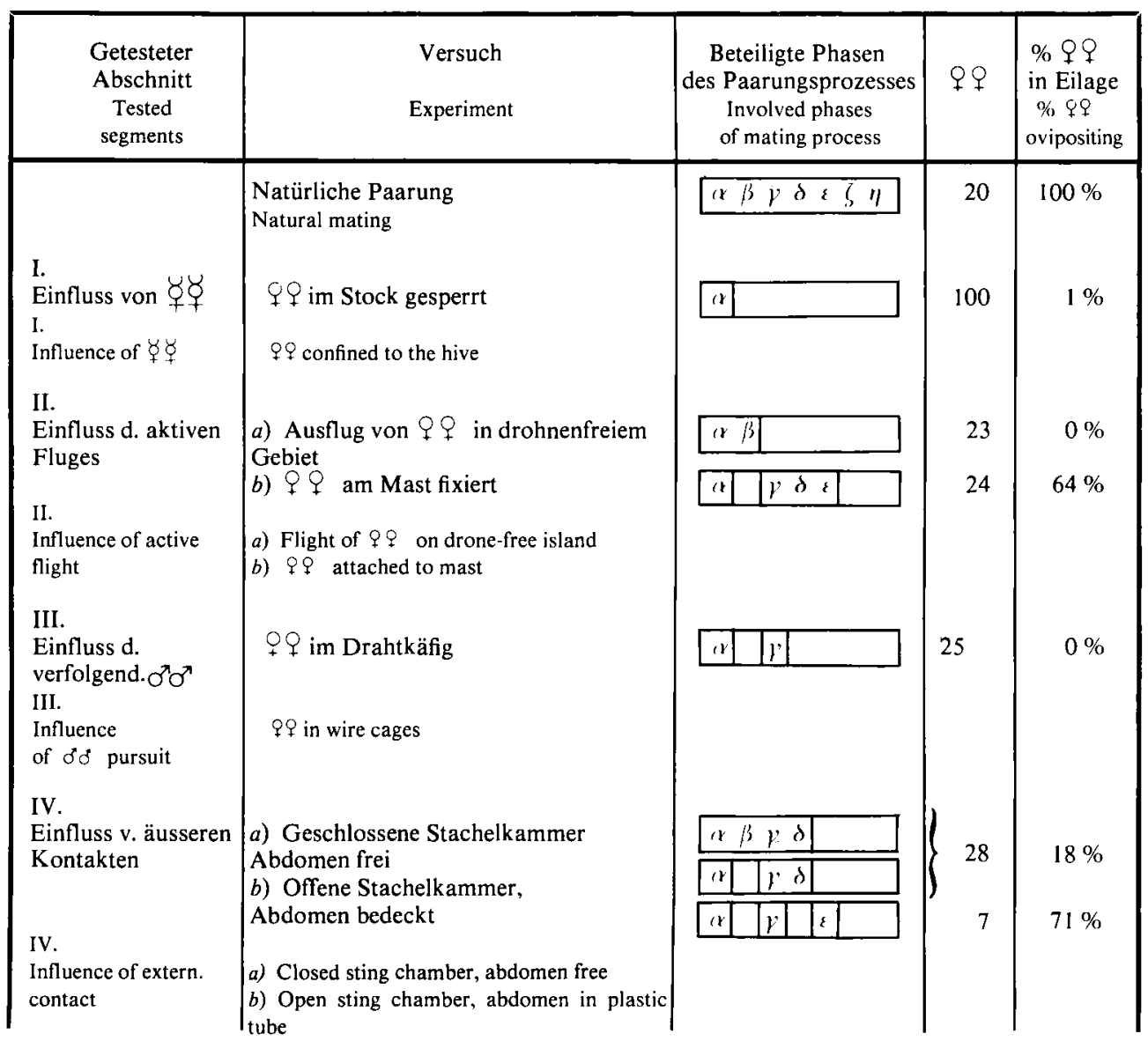




\begin{tabular}{|c|c|c|c|c|}
\hline $\begin{array}{l}\text { Getesteter } \\
\text { Abschnitt } \\
\text { Tested } \\
\text { segments }\end{array}$ & $\begin{array}{l}\text { Versuch } \\
\text { Experiment }\end{array}$ & $\begin{array}{l}\text { Beteiligte Phasen } \\
\text { des Paarungsprozesses } \\
\text { Involved phases } \\
\text { of mating process }\end{array}$ & n $q$ & $\begin{array}{l}\% q q \\
\text { in Eilage } \\
\% 9 q \\
\text { ovipositing }\end{array}$ \\
\hline $\begin{array}{l}\text { V. } \\
\text { Einfluss d. } \\
\text { Kontakte in } \\
\text { Stachelkammer } \\
\text { und Bursa cop. }\end{array}$ & $\begin{array}{l}\text { a) Direkter Kontakt mit halb } \\
\text { evertiertem Endophallus von aktiven } \\
\delta \delta \text { (II b und IV b) } \\
\text { b) Direkter Kontakt mit halb } \\
\text { evertiertem Endophallus von toten } \delta 8 \\
\text { im Labor } \\
\text { c) Kontakt durch ein Nylongewebe } \\
\text { behindert (KoENIGER, G. 76) } \\
\text { a) Direct contact with semi-everted endo- } \\
\text { phallus of active } \delta^{\circ} \delta^{\circ} \text { (II b + IV b) } \\
\text { b) Direct contact with semi-everted endo- } \\
\text { phallus of dead } \sigma^{\circ} \text { (manual) } \\
\text { c) Contact through a nylon fabric }\end{array}$ & 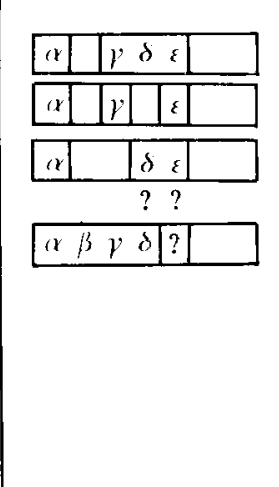 & 16 & $\begin{array}{r}68 \% \\
4 \% \\
69 \%\end{array}$ \\
\hline $\begin{array}{l}\text { VI. } \\
\text { Einfluss d. }\end{array}$ & Injektion von Homogenisat, frischem & & & \\
\hline $\begin{array}{l}\text { Sekrete d. männl. } \\
\text { Anhangsdrüsen }\end{array}$ & $\begin{array}{l}\text { Mucus oder Teilen des Endophallus } \\
\text { a) in die Körperhöhle } \\
\text { b) in die Stachelkammer und Bursa cop. }\end{array}$ & \begin{tabular}{|l|}
$\alpha$ \\
$\alpha$ \\
\end{tabular} & $\begin{array}{l}21 \\
78\end{array}$ & $\begin{array}{l}5 \% \\
8 \%\end{array}$ \\
\hline $\begin{array}{l}\text { VI. } \\
\text { Influence of the } \\
\text { secretions from male } \\
\text { acces. glands }\end{array}$ & $\begin{array}{l}\text { Injection of homogenate or fresh mucus into } \\
\text { a) In the body cavity } \\
\text { b) The sting chamber and bursa cop. } \\
\text { (KoENIGER, G. 1976) }\end{array}$ & & & \\
\hline $\begin{array}{l}\text { VII. } \\
\text { Einfluss d. Spermas } \\
\text { VII. } \\
\text { Influence of sperm }\end{array}$ & $\begin{array}{l}\text { Künstliche Besamung ohne } \\
\mathrm{CO}_{2} \text {-Narkose (MACKENSEN, 1947) } \\
\\
\text { Artificial insemination without } \mathrm{CO}_{2} \\
\text { narcosis (MACKENSEN, 1947) }\end{array}$ & \begin{tabular}{|l|}
$\alpha$ \\
\end{tabular} & 34 & $0 \%$ \\
\hline
\end{tabular}

Nachdem sich die Flugaktivität der Königin als wenig bedeutend erwiesen hatte, mussten die verschiedenen Kontakte mit den Drohnen systematisch untersucht werden. Der rein visuelle und olfaktorische Kontakt blieb ohne Wirkung (Tab.6, III). Der spezifische Kontakt zwischen den männlichen und weiblichen Genitalorganen wurde mit verschiedenen Methoden untersucht :

1. Bei zwei Versuchsgruppen war der Kontakt zwischen den Genitalorganen vollständig verhindert (siehe Kapitel $2 b$, Tab. 6, IV $a$ ).

2. Der direkte Kontakt zwischen Endophallus, Bursa copulatrix und Stachelkammer wurde durch ein mit Klebstoff getränktes Nylongewebe behindert (KoENIGER G., 1976 - Tab. 6, V b). 
3. Die Eversion des Endophallus in die Stachelkammer und Bursa copulatrix blieb unvollständig, es wurde wenig oder kein Sperma und Mucus in die Königin übertragen (siehe Kapitel $1 b, 2 c$ und 3 - Tab. 6, IV $b$ und V $c$ ).

4. Nur die männlichen Sekrete des vollständig evertierten Endophallus wurden in die Königin übertragen (KoenIGer G., 1976 - Tab. 6, VI).

Von den Königinnen, bei denen der Genitalkontakt vollständig verhindert war, begannen nur $16 \%$ mit der Eiablage. Waren die Stachelkammern dagegen offer, begannen $70 \%$ der Königinnen Eier zu legen, auch bei der Versuchsreihe, bei der die Stachelkammern durch das Nylongewebe abgedeckt waren. Bei diesen Experimenten waren die Drohnen selbst aktiv und die Kontakte fanden auf dem Sammelplatz statt.

Die Versuche im Labor, die Kontakte nachzuahmen, führten zu keiner nennenswerten Erhöhung der Eiablage. Aus der Zeit der Entwicklung der künstlichen Besamung gibt es einige Angaben über erfolgreiche Handbesamungen (LAIDLAW 1932 und 1944, MACKENSEN und RoBerts 1948, Watson 1927). Da aber im allgemeinen bei diesen Versuchen mit älteren Königinnen unter Narkose gearbeitet wurde, helfen diese Ergebnisse bei der Klärung der Induktion der Eiablage nicht weiter.

Abschliessend können wir feststellen, dass die Induktion der Eiablage noch nicht restlos gaklärt ist, aber es gelang nachzuweisen, dass nicht alle Phasen des Paarungsablaufes dabei beteiligt sind. So hatten weder der aktive Flug noch das Begattungszeichen einen entscheidenden Einfluss. Auch die äusseren Kontakte spielen wohl nur eine untergeordnete Rolle. Ein entscheidender Reiz muss von einem bis zur Cervix evertierten Endophallus ausgehen und im Bereich Stachelkammer - Bursa copulatrix perzipiert werden. Welche Art von Reizen dabei ausschlaggebend ist, soll in weiteren Versuchen geprüft werden.

\section{SUMMARY}

IN WHICH SEGMENT OF THE MATING PROCESS OF THE QUEEN BEE DOES THE INDUCTION OF OVIPOSITION OCCUR?

The mating process was divided artificially into a series of experiments. In this way the significance of definite steps triggering oviposition could be evaluated either by testing one phase in isolation or, conversely, by omitting just this phase in the mating process.

Since it was known that neither the filling of the spermatheca with sperm (MACKENSEN, 1947) nor the transfer of mucous or a homogenate of the fully everted endophallus into the sting chamber and bursa copulatrix (KoENiger G., 1976) triggered oviposition, the influence of flight and different degrees of contact with drones was now tested.

In all experiments the age of the queens was between 10 and 14 days. Each day the colonies were checked for eggs. When the queens were 21-23 days old, the ovaries were measured. 
A. Influence of active flight of the queens

a) Active flight without contact with drones

The queens were allowed to fly on an island from which all drones had been removed. None of the queens started oviposition. The weight of ovaries was the same as in queens confined to their hives (Table 1).

b) Mating without active flight

The queens were fixed on a thin wire and the sting chamber was glued open (Fig. 1). In this position queens normally will not fly, but the drones can mate actively (in free flight). In all experiments with fixed queens the eversion of the endophallus stopped half way, so that nearly no mucous or sperm could be transferred into the queen. After 6-8 of these incomplete insertions (matings) $64 \%$ of the queens started to lay eggs (Table 2).

\section{B. Influence of different kinds of contact with drones}

a) Visual and olfactory contact

Queens, confined in a small wire cage, were brought to a drone congregation area, where swarms of drones pursued each queen for about 15 minutes. No queen started oviposition.

b) External contact

1. External contact possible, but insertion of endophallus prevented

The sting chamber of the queens was completely closed by gluing tergite and sternite together. Some of the queens could fly freely, others were fixed on the mast. Of a total of 28 queens 5 started oviposition (Table 3).

2. External contact prevented, insertion of the endophallus possible

The abdomen of the queens was covered by a plastic tube, the sting chamber being kept open (Fig. 2). In this way the drones could mate, but without touching the queen externally. Of 7 mated queens 5 started oviposition (Table 4).

3. Influence of manual insertion of the half everted endophallus into the sting chamber

The sting chamber was held opened with glue. The endophalli of drones were everted either by using chloroform or by pressing the thorax. Then the half everted endophalli of 10 drones were inserted by hand into the sting chamber of each queen. This treatment did not result in a tight and strong connection as observed in the experiments on the mast with freely flying drones. Only one of 24 queens laid eggs (Table 5).

All data known on this problem are summarized in Table 6. A high percentage of the queens whose sting chambers were open during the experiments started ovipositing. All factors such as active flight or external contact with drones showed none or little effect on oviposition. Thus the perception of the stimulus for oviposition must occur in the sting chamber and bursa copulatrix, but is independant of the mating sign.

\section{RÉSUMÉ}

\section{DANS QUELLE PHASE DU COMPORTEMENT D'ACCOUPLEMENT} DE LA REINE D'ABEILLE A LIEU L'INDUCTION DE LA PONTE?

Au ccurs d'une série d'expériences on a divisé artificiellement le processus d'accouplement en plusieurs phases. De cette façon on a pu contrôler l'importance de phases précises dans l'induction de la pon te, soit en testant une phase isolément, soit en l'omettant dans le déroulement du processus. 
Puisque l'on sait que ni le remplissage de la spermathèque par le sperme (MACKENSEN, 1947), ni le transfert du mucus ou d'une partie de l'endophallus en totale éversion dans la chambre de l'aiguillon et la bursa copulatrix n'induisent la ponte, on a voulu tester l'influence du vol et de divers degrés de contact avec les mâles.

Dans toutes les expériences, les reines étaient âgées de 10 à 14 jours. Chaque jour on a vérifié l'absence ou la présence d'œufs dans les colonies. Lorsque les reines ont atteint 21 à 23 jours, leurs ovaires ont été mesurés.

\section{A. Influence du vol actif des reines}

a) Vol actif sans contact avec les mâles

On a laissé voler des reines sur une île dont tous les mâles avaient été supprimés. Aucune des reines ne se mit à pondre. Le poids des ovaires était le même que chez des reines confinées dans leur ruche (tableau 1).

\section{b) Accouplement sans vol actif}

Les reines ont été fixées à un mince fil de fer et la chambre de l'aiguillon maintenue ouverte à l'aide de colle (fig. 1). Dans cette position les reines ne pouvaient pas voler, mais les mâles pouvaient copuler activement (en vol). Dans toutes les expériences avec des reines attachées, l'éversion de l'endophallus s'est arrêtée à mi-chemin, si bien que presque aucun mucus ni sperme n'a pu pénétrer dans la reine. Après 6 à 8 de ces copulations incomplètes, $64 \%$ des reines se mirent à pondre (tableau 2).

\section{B. Influence de diverses sortes de contact avec les mâles}

a) Contact visuel et olfactif

Des reines, enfermées dans une petite cage grillagée, furent portées sur un lieu de rassemblement de mâles, où des essaims de mâles poursuivirent chaque reine pendant $15 \mathrm{mn}$. Aucune reine ne se mit à pondre.

\section{b) Contact externe}

1. Contact externe possible, mais pénétration de l'endophallus impossible.

La chambre de l'aiguillon des reines a été complètement fermée en collant le tergite et le sternite l'un contre l'autre. Certaines reines pouvaient voler librement, d'autres étaient fixées à un mât. Sur 28 reines 5 se mirent à pondre (tableau 3).

2. Pas de contact externe, pénétration de l'endophallus possible.

L'abdomen des reines a été entouré d'un tube plastique, la chambre de l'aiguillon restant ouverte (figure 2). De cette cette manière les mâles pouvaient copuler mais sans toucher la reine extérieurement. Sur 7 reines s'étant accouplées, 5 se mirent à pondre (tableau 4).

3. Influence de la pénétration manuelle de l'endophallus en semi-éversion dans la chambre de l'aiguillon.

La chambre de l'aiguillon a été maintenue ouverte à l'aide de colle. On a provoqué l'éversion des endophallus des mâles soit par le chloroforme, soit par pression sur le thorax. Ensuite on a introduit à la main les endophallus en semi-éversion de 10 mâles dans la chambre de l'aiguillon de chaque reine. Ce procédé n’a pas abouti à un rapport étroit, comme cela avait été le cas lors des expériences sur le mât avec des mâles volant librement. Une seule reine sur 24 a pondu (tableau 5).

Toutes les données connues concernant ce problème sont résumées dans le tableau 6 . Un fort pourcentage de reines dont les chambres de l'aiguillon furent ouvertes pendant les expériences commencèrent à pondre. Tous les facteurs tels que vol actif ou contact externe avec les mâles ont eu pas ou peu d'influence sur la ponte. La perception du stimulus de la ponte doit donc se situer dans la chambre de l'aiguillon et la bursa copulatrix, mais elle est indépendante des signes d'accouplement. 


\section{LITER ATUR VERZEICHNIS}

Alber M., Jordan R., Ruttner F., Ruttner H., 1955. - Von der Paarung der Honigbiene. Z. Bienénkunde, 3; 1-28.

KoENIGER G., 1976. - Einfluss der Kopulation auf den Beginn der Eiablage bei der Bienenköningin (Apis mell. L.). Apidologie 7; 343-355.

Koeniger G., Koeniger N., Fabritius M., 1979. - Some detailed observations of mating in the honeybee. Bee World, 60; 53-57.

LaidLAW H. H., 1932. - Hand mating of queenbees. Amer. Bee Journal, 72; 286.

Laidlaw H. H., 1944. - Artificial irsemination of the queen bee (Apis mell. L.). J. Morph., 74; 429-465.

MACKENSEN O., 1947. - Effect of carbon dioxide on initial oviposition of artificially inseminated and virgin queen bees. J. Econ. Ent., 40; 344-349.

MACKENSEN O. and RoberTs W. C., 1948. - A manual for the artificial insemination of queen bees. U.S.D.A. Bull. Ent. and Plant Quar. ET - 250.

PeER D. F., 1957. - Further studies on the mating range of the honeybee Apis mell. L. Canad. Entomol, 89; $108-110$.

Renner M. und Vierling G., 1977. - Die Rolle des Taschendrüsenpheromons beim Hochzeitsflug der Bienenkönigin. Behav. Ecol. Sociobiol., 2; 329-338.

Watson L. R., 1927. - Controlled mating of queenbees. Hamilton, III., Condensed in Amer. Bee J., 67/5, 6, 7; 235-236, 300-302, 364-365. 